\title{
A Robust Technique for Image Mosaicing using Modified SIFT
}

Jasmeet Kaur

School of Electronics Engineering, Lovely Professional University, Phagwara, Punjab, India;

pahwa.jasmeet@gmail.com

\begin{abstract}
In this paper a robust technique is used for image mosaicing to reduce the computational time and increase the efficiency through modified Scale Invariant Feature Transform SIFT. Modified Scale Invariant Feature Transform algorithm is used to increase the efficiency and to reduce the computational time. In this normalized cross correlation is used to find the best possible match for the image warping. Area found by Normalized Cross Correlation is used for feature matching, through this method computational time is reduced. Two different methods are combined to get the best output. As the number of matches increased the efficiency of the algorithm also increased. The area for matching is reduced so the computational time gets reduced. The output mosaicked image is warped by the best possible matches This paper depicts the implementation of the real images click by a normal Samsung phone camera at different angles and locations. Homography is used to find the angular relation between the images. Normalized Modified SIFT algorithm is used to increase the efficiency and reduce the computational time. Mosaiced image is efficient enough as compare to SIFT algorithm.
\end{abstract}

Keywords: Computational Time, Efficiency, Image Mosaicing, Modified Scale Invariant Feature Transform (SIFT) Algorithm, Normalized Cross Correlation

\section{Introduction}

Image mosaicing is a technique in which we are trying to increase the field of view of our image. We are not able to click images with large field of view with our normal standard cameras because they have limited specifications in it. So to increase our field of view either we need to buy a special lens or cameras or by applying different techniques on it. Buying a special kind of camera is bit costlier, so it is easy to apply different techniques of image mosaicing on different images to make it a panoramic view $\frac{1,7,10}{}$.

The most important step in image mosaicing is image matching. Image matching can be done by two methods: pixel based matching and feature based matching. The pixel based matching has high complexity so we prefer only feature based matching. It has different steps: feature detection, feature matching, transformation and appending images. For feature detection we use Scale Invariant Feature Transform (SIFT) technique. After detecting the features, we match those features to append different images $\frac{2,11}{}$.

Every process or technique has some limitations so SIFT have. It has high complexity and computational time due to which it takes lot of time to process and difficult to understand. To reduce this limitation, we start research on this topic and find optimal solution to reduce these effects. In this report we apply five different methods to improve the quality of matching. And compare these methods we time and feature parameters for analyzing that which one is better technique and easy to work with ${ }^{3.4}$

The basic technique is SIFT algorithm introduced by David Lowe in 1994. By doing some advancement in this basic process we get a better approach for image matching. To reduce the limitations, we add normalized cross correlation so that we can reduce the time parameter and increase the number of matching features and also we normalized the gradient factor for better results ${ }^{5}$.

Different steps involved in SIFT algorithm are: 
i. Scale space extrema detection: First step is to calculate all the features in the image at each scale. These features can be calculated by calculating the difference of Gaussian. Difference of Gaussian scale space was proposed to generate the following output using different scale kernel of difference of Gaussian to convolution with images to generate:

$$
\begin{aligned}
& D(x, y, \delta)=(G(x, y, k \delta)-G(x, y, \delta)) * I(x, y) \\
& =L(x, y, k \delta)-L(x, y, \delta)
\end{aligned}
$$

Where

$$
G(x, y, \delta)=\frac{1}{2 \pi \delta} e^{-\frac{x^{2}+y^{2}}{2 \delta^{2}}}
$$

$\delta$ is scale coordinates and $(\mathrm{x}, \mathrm{y})$ is spatial coordinates. ii. Key point localization: In this step we remove those points or features that have very less or poor contrast. This can be done by calculating Laplacian. The location is given as:

$$
z=\frac{\partial^{2} D^{-1} \partial D}{\partial^{2} x^{2} \partial x}
$$

Where $\mathrm{z}$ is the location. If the value at $z^{\text {th }}$ location is below thresholding then that point is excluded.

iii. Orientation assignment: All the different orientations are applied to the image in this step only. It includes scaling, translation, etc. orientations are applied to the key locations only.

$$
\begin{aligned}
& m(x, y)=\sqrt{\begin{array}{l}
(L(x+1, y)-L(x-1, y))^{2}+ \\
(L(x, y+1)-L(x, y-1))^{2}
\end{array}} \\
& \theta(x, y)=\tan ^{-1}\left(\frac{L(x, y+1)-L(x, y-1)}{L(x+1, y)-L(x-1, y)}\right)
\end{aligned}
$$

iv. Keypoint descriptor: The local gradient data used above is used to create a keypoint descriptor. The gradient information is combined with the orientation data ${ }^{\frac{13}{3}}$.

v. From both the images key-points descriptors are compared. Key-points having high similarity are registered as matching points.

The resulting vectors are known as SIFT keys are mostly and widely used to find possible objects in a nearest neighbour approach $\frac{12,8}{\text {. }}$.
Figure 1 shows the flow chart of steps involved in SIFT algorithm. After applying these steps, we append the images by finding the matching points and there are different algorithms to append the images. In this report we apply Random Sample Consensus (RANSAC) algorithm and homography for image blending.

RANSAC algorithm is a robust technique for fitting of templates containing many outliers. RANSAC algorithm is a non-deterministic algorithm because a reasonable result with certain probability is generated, probability increases as the number of iterations increases. RANSAC algorithm is an iteration based algorithm. This algorithm is basically used to find the points in the space with known locations.

There are some assumptions like the data should be consists of inliers. Inliers are the distribution of data that can be specified by some template or model parameters. RANSAC algorithm has composed of two steps:

1. A sample subset is selected which consists of randomly selected minimal data items from the input dataset. From these dataset model parameters are selected.

2. After selecting the model parameters, algorithm applied to the input. It will check inliers and the outliers means which data is consistent to the given model parameters and which are not. The dataset which are consistent are the inliers and which are not consistent are the outliers.

Inliers set is the consensus set. This algorithm repeat itself unless we will get enough inliers.

Steps to perform RANSAC algorithm:

Step 1: Detect features.

Step 2: Calculate matches.

Step 3: Apply

- Select minimal data items.

- Compute model parameters.

- Determine inliers.

Step 4: Compute matches based on all inliers.

Step 5: Check for additional matches.

In mosaicing, RANSAC is a robust approach, that's why we use RANSAC algorithm to make our algorithm more efficient and robust.

Homography is a projective plane transformation through which we can find the relation between two images having same planar surface but taken from different viewpoints? ${ }^{9}$ Hence homography is used for registering images having similar planar properties. To 
perform homography, some estimation should be calculated through the detected points at different coordinates in different images but real time location is at the same point. The most robust technique used to get the detected is RANSAC algorithm. So to register the two images in this paper RANSAC and homography is used.

By calculating the points in RANSAC algorithm, homography can be estimated and according to that changes has to be made to register two images.

\section{Proposed Work}

In this method normalized gradients are used in place of normal gradients to reduce the complexity and computational time. Normalized cross correlation is added to reduce the computational time and complexity. Read the different images and apply normalized cross correlation. And for matching feature points Euclidean distance is used and only those features are taken as matched features

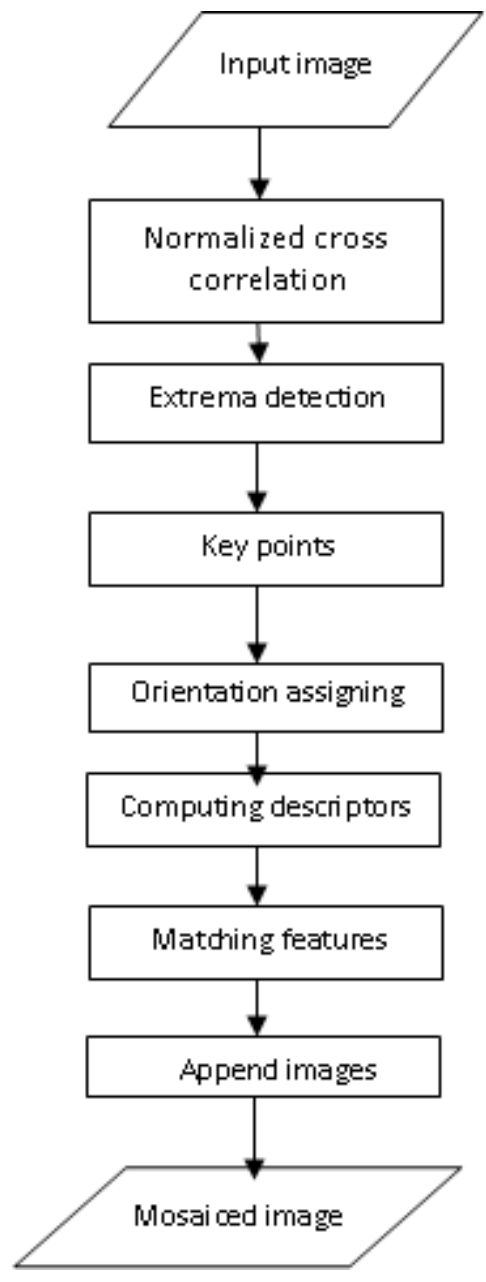

Figure 1. Steps to perform SIFT algorithm whose difference is less than 0.6 as defined. We can adjust this depending upon whether we want more matching or less matching. If we decrease this distance or thresholding value, then less number of matching will occur whereas if we increase this value unwanted matching will also occur.

After calculating the features, we append the images side by side for feature matching ${ }^{14}$. When the matching occurs we mosaiced the images by homography. We get better results for homography because in this less ghosting effect occurs than other registering methods.

Time taken for all this is less than the normal SIFT algorithm. So it is better than the normal or basic SIFT algorithm.

\section{Experimental Results}

Figure 2 and 3 are the input images. These images are taken from Samsung camera and the location is Lovely professional university ${ }^{15}$.

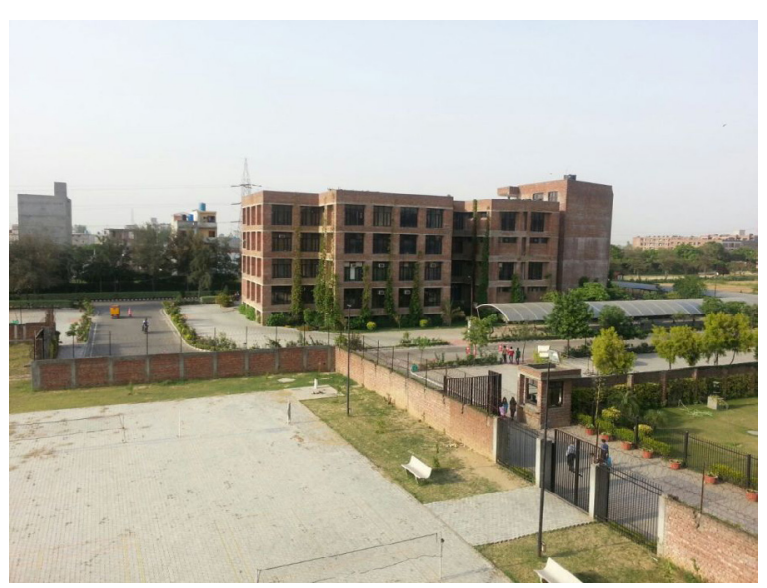

Figure 2. First input image

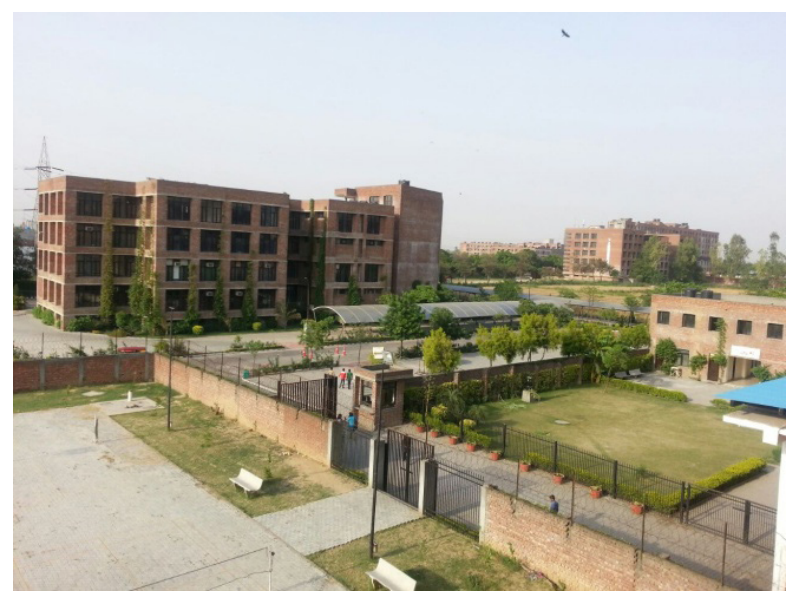

Figure 3. Second input image 
The method firstly applies the modified SIFT algorithm. In which normalized cross correlation is used and then SIFT algorithm with normalized gradient is used that gives the features detected by this algorithm. Figure 4 shows the matching points. There are 1307 matching points and 1189 are unique matching points. The efficiency of this algorithm is $90.97 \%$ whereas the efficiency of the algorithm without using normalized cross correlation is $90.82 \%$. In modified SIFT algorithm the total matches found are 1296 and unique matches are 1177.

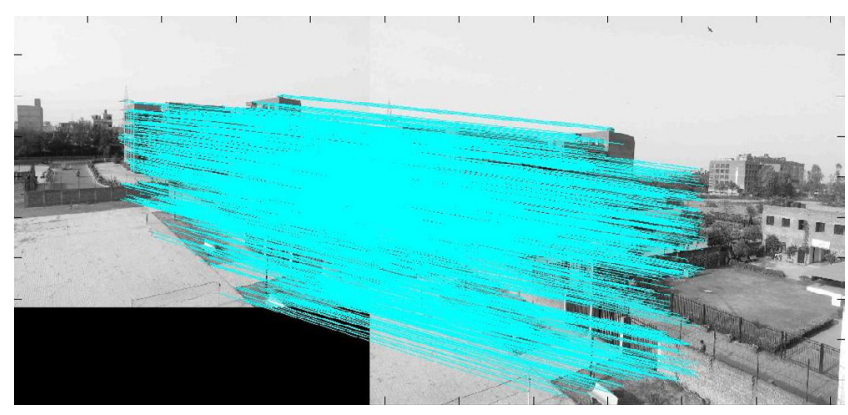

Figure 4. Matching points

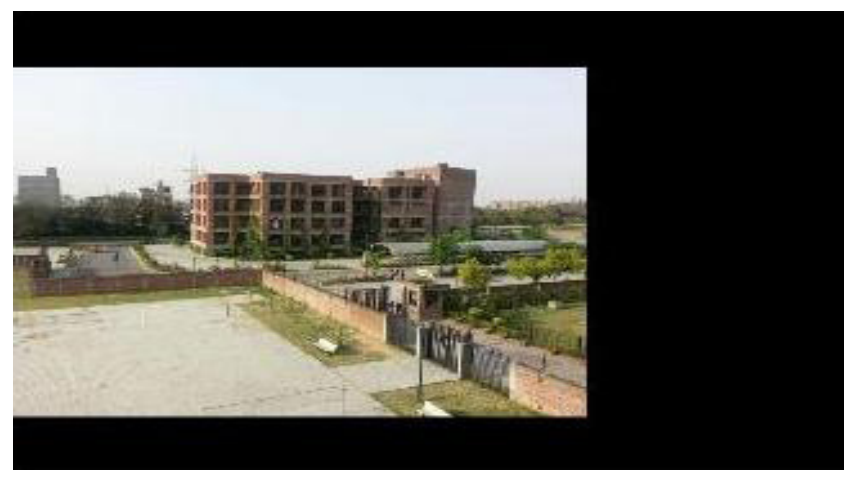

Figure 5. Transformation applied on image1

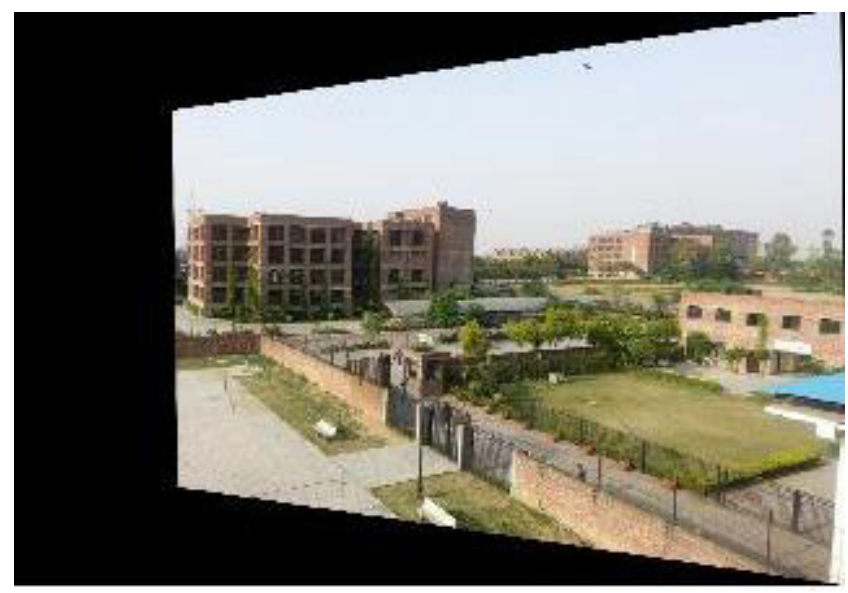

Figure 6. Transformation applied on image 2
Homography is applied to find the transformation that what kind of changes need to do to create a mosaiced image. Figure5 and Figure6 are the homography output images. After Image warping, figure7 is the output mosaicked image.

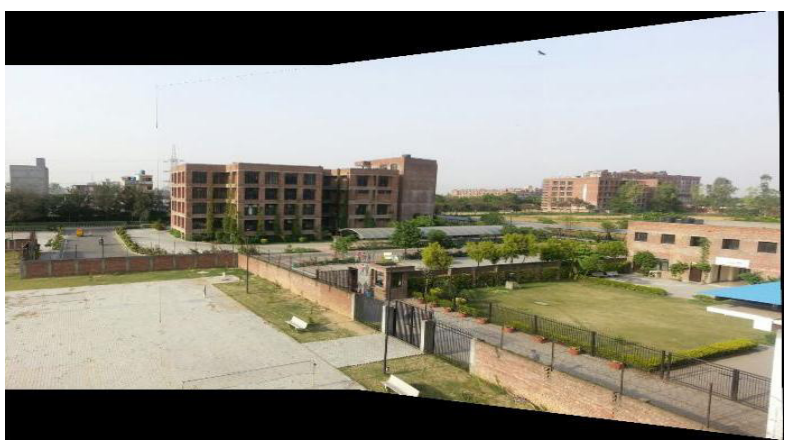

Figure 7. Mosaiced image

Table 1. Comparison between two methods

\begin{tabular}{|l|l|l|l|}
\hline \multirow{2}{*}{ Different methods } & \multicolumn{3}{|l|}{ Parameters } \\
\cline { 2 - 4 } & $\begin{array}{l}\text { Time } \\
\text { (seconds) }\end{array}$ & $\begin{array}{l}\text { Matching } \\
\text { features }\end{array}$ & Unique \\
\hline Modified SIFT & 22.388 & 1296 & 1177 \\
\hline This paper Result & 17.037 & 1307 & 1189 \\
\hline
\end{tabular}

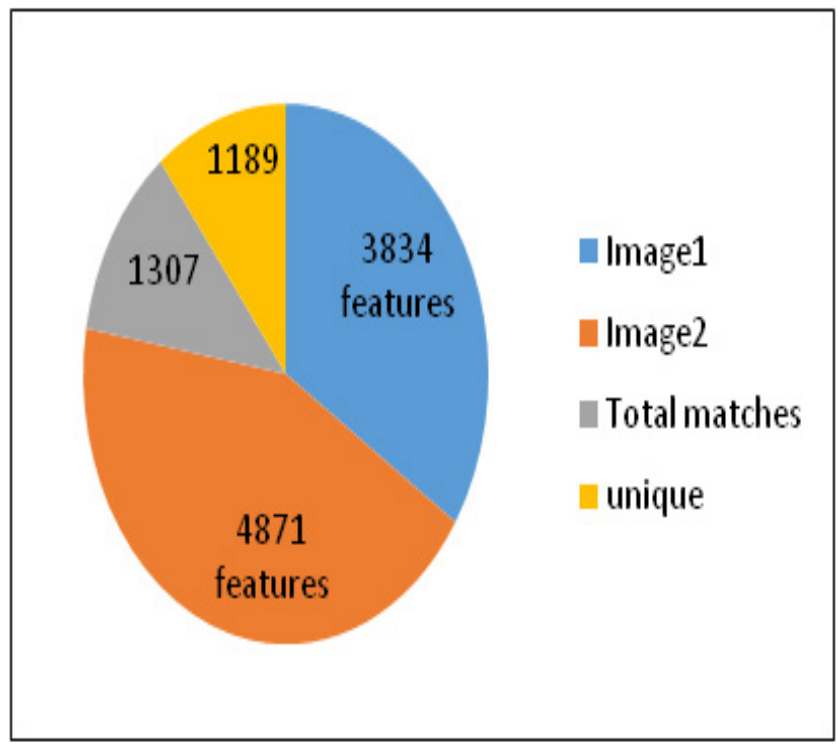

Pie chart for feature matching in modified SIFT algorithm

\section{Conclusion}

In this paper, modified SIFT algorithm using normalized cross correlation is used. As comparing the results with 


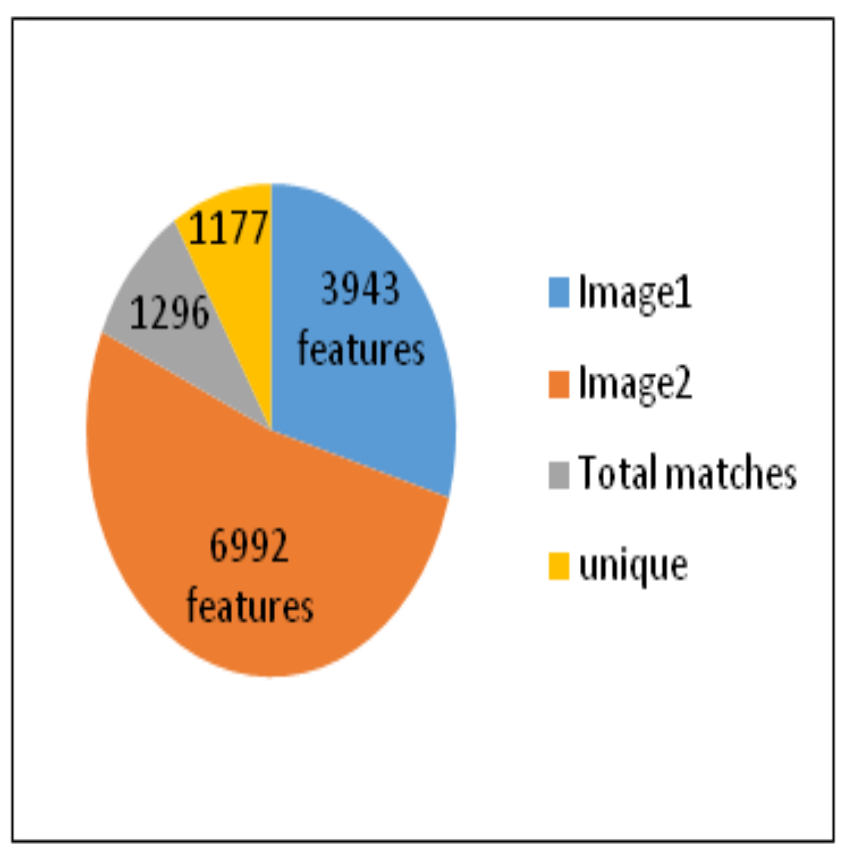

Pie chart for feature detection using this algorithm

simple modified SIFT algorithm the computational time is less and more features are detected and matched using this algorithm. RANSAC and Homography algorithms are used to reduce the influence of wild points and for appending the images to get the mosaiced view. The efficiency of this paper is $90.97 \%$. Future work can be done by implementing this algorithm for satellite images and videos ${ }^{6}$.

\section{References}

1. Shmuel P, Joshua H. Panoramic Mosaics Manifold Projection. IEEE Trans. on Panoramic Mosaicing. 1997, p. 338-43.

2. Yining D, Tong Z. Generating panorama photos, HewlettPackard Labs.

3. Seong H, Hyung K, Sang L, Nam C, Soo K. Panorama mosaic optimization for mobile camera systems. IEEE Trans. on Panorama, 2007.
4. Alex R, Glora E, Shmuel P. Minimal Aspect Distortion mosaicing of long scenes. Springer, 22 November 2007, 187-206.

5. Lin Z, Shengping Z, Junn Z, Yunhu Z. Dynamic image mosaic via sift and dynamic programming. Springer, 19 September 2013.

6. Zezzhong X. Consistent image alignment for video mosaicing. Springer. 16 February 2011, 129-35.

7. Akifumi I. Image mosaicing: create high quality panoramic multi spectral image. 12 February 2002.

8. Lin J, Wang Y, Liang H. Image mosaic based on simplified SIFT"IERI, 2012, v.10.

9. Pengrui Q, Ying L, Hui R. Image mosaics algorithm based on SIFT feature point matching and transformation parameters automatically recognizing. Proceedings of the 2nd International Conference on Computer Science and Electronics Engineering (ICCSEE 2013), 2013. Doi:10.2991/ iccsee. 2013.392

10. Yoav S, Shree N. Generalized mosaicing: high dynamic range in a wide field of view. International Journal of Computer Vision. 2003 Jan; 28:245-67.

11. Matthew Brown, David G. Lowe. Recognising Panoramas. Department of Computer Science, University of British Columbia. International Conference on Computer Vision (ICCV 2003), Nice, France, 2003 Oct, p. 1218-25

12. Xingxing $S$, Wenxing $B$. The remote sensing image matching algorithm based on the normalized cross-correlation and SIFT. Springer. 10 December 2013, p. 417-22.

13. David G. Lowe. Object recognition from local scale-invariant features. Department of Computer Science, University of British Columbia. Proc. of the International Conference on Computer Vision, Corfu, 1999 Sept, p. 1-8.

14. Matthew Brown, David G. Lowe. Automatic panoramic image stitching using invariant features. Department of Computer Science, University of British Columbia. International Journal of Computer Vision. 2007 Aug; 74(1):59-73

15. Sai Venu PK, Jilani SAK, Ramana Reddy P.. A Real-Time Image Mosaicing using Scale Invariant Feature Transform. Indian Journal of Science and Technology. 2016 Mar; 9(12). DOI: $10.17485 /$ ijst/2016/v9i12/88175. 